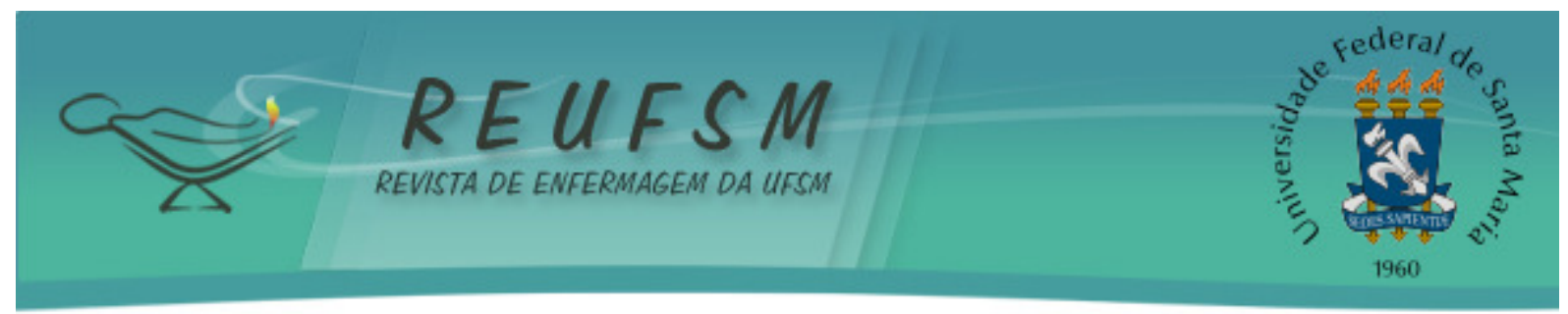

ARTIGO ORIGINAL

\title{
PERCEPÇÃO DOS TRABALHADORES DE ENFERMAGEM QUANTO A BIOSSEGURANÇA NO CUIDADO QUIMIOTERÁPICO
}

\author{
PERCEPTION OF NURSING WORKERS ABOUT THE BIOSECURITY ON \\ CHEMOTHERAPY CARE
}

\section{PERCEPCIÓN DE LOS TRABAJADORES ENFERMERÍA SU BIOSEGURIDAD EN EL CUIDADO EN QUIMIOTERAPIA}

\author{
Wendel Mombaque dos Santos ${ }^{1}$ \\ Ana Paula Scheffer Schell da Silva ${ }^{2}$ \\ Luísa Ricardo Netto ${ }^{3}$
}

Doi: $10.5902 / 217976928531$

RESUMO: Objetivo: identificar a percepção dos profissionais de Enfermagem quanto a biossegurança no ambiente de cuidado quimioterápico. Método: trata-se de um estudo de caso exploratório-descritivo realizado com profissionais da Enfermagem de um serviço de quimioterapia. Os dados foram coletados por meio de observação não participante e entrevista semiestruturada, e analisados com auxílio da Análise de Conteúdo. Resultados: emergiram duas categorias: segurança do profissional em ambiente quimioterápico e segurança do paciente em ambiente quimioterápico. As práticas adotadas na manipulação de antineoplásicos expõem os profissionais ao risco de contato com drogas e inalação de partículas aerossolizadas, favorecendo o surgimento de doenças e/ou agravos ao trabalhador. Considerações Finais: os profissionais percebem os riscos a que estão expostos, entretanto não há adesão a medidas de proteção. Há necessidade de conscientizar os profissionais quanto às medidas de segurança na administração de quimioterápicos, de forma a garantir qualidade e segurança no cuidado prestado aos pacientes.

Descritores: Enfermagem; Saúde do trabalhador; Riscos químicos; Biossegurança; Antineoplásicos.

ABSTRACT: Aim: to identify the perception of nursing professionals working in an oncology unit regarding safety in the environment of care chemotherapy. Method: this was a case study with descriptive exploratory which was performed with a professional nursing oncology service. Data were collected through non-participant observation semistructured interviews and analyzed using analysis of Content. Results: two categories emerged: patient safety in chemotherapy environmental and safety professional in the chemotherapy room. The practices in handling antineoplastic expose professionals to the risk of contact with drugs and inhalation of aerosolized particles, favoring the emergence of diseases and/or injuries to nursing work. Conclusions: professionals understand the risks they are exposed to, however there is no adherence to protective measures for reducing these. Contact the necessity of continuing education about the safety measures in the administration of chemotherapy, to ensure quality and safety in the care provided to patients.

1 - Enfermeiro. Especialista em Ciências da Saúde e em Enfermagem do Trabalho. Universidade Federal do Pampa. Uruguaiana/RS, Brasil. E-mail: wendelmombaque@hotmail.com

2 - Enfermeira. Doutora. Professora Adjunta do Departamento de Enfermagem da Universidade Federal de Ciências da Saúde de Porto Alegre (UFCSPA). Porto Alegre/RS, Brasil. E-mail: anaschell@gmail.com

3 - Enfermeira. Especialista em Enfermagem Oncológica. Hospital Doutor Bartholomeu Tacchini. Bagé/RS, Brasil. E-mail: luisarnetto@hotmail.com 


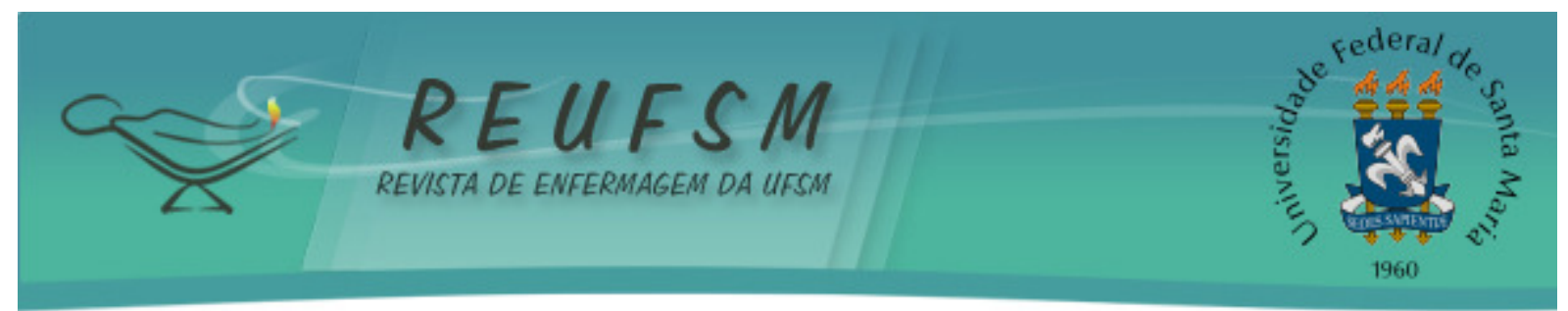

Descriptors: Nursing; Occupational health; Chemical Contamination; Biosecurity; Antineoplastic Agents.

RESUMEN: Objetivo: identificar la percepción de enfermeros sobre bioseguridad en ambiente de cuidado quimioterápico. Método: estudio exploratorio descriptivo que se realizó en un servicio de quimioterapia. Los datos fueron recolectados a través de observación no partipante y entrevistas semiestructuradas, analizados mediante Análisis de Contenido. Resultados: surgieron dos categorías: seguridad del profesional en ambiente quimioterápico y seguridad del paciente en ambiente quimioterápico. Las prácticas adoptadas en la manipulación de antineoplásicos exponen a los profesionales al riesgo de contacto con drogas e inhalación de partículas en suspensión por aerosol, favoreciendo la aparición de enfermedades y/o lesiones. Consideraciones finales: los profesionales comprenden los riesgos a que están expuestos, pero no hay adhesión a las medidas de protección. Es necesaria la concienciación de los profesionales sobre medidas de seguridad en administración de quimioterápicos, para garantizar la calidad y seguridad prestada a los pacientes.

Descriptores: Enfermería; Salud laboral; Contaminación química; Bioseguridad; Antineoplásicos.

\section{INTRODUÇÃO}

O câncer é constituído de um conjunto que envolve mais de 200 doenças distintas com variedade de causas e história natural. Foi descrito e definido por Hipócrates, em 500 a.C. na Grécia, como uma enfermidade de mau prognóstico. ${ }^{1}$

0 tratamento dessa doença consiste na existência de diversos tipos de condutas, a destacar o uso de agentes antineoplásicos ou quimioterápicos, combinados ou isolados. Estes são medicamentos que atuam no nível sistêmico com a finalidade de inibir o crescimento e/ou a disseminação de tumores por meio da destruição das células malignas. Outros tratamentos incluem a radioterapia, iodoterapia, tratamento cirúrgico, transplante de células-tronco hematopoéticas e bioterapia que consiste no uso de agentes derivados de fontes biológicas. ${ }^{2-4}$

Mesmo com a utilização dos tratamentos disponíveis, é crescente o número de óbitos de pacientes portadores de câncer. Anualmente, nos Estados Unidos da América, estima-se mais de 580 mil mortes que tem como causa o câncer. A magnitude do câncer, como problema de saúde pública mundial, é bem conhecida e vem ocupando, no Brasil, as primeiras posições em termos de mortalidade. ${ }^{5-6}$

As experiências pessoais e profissionais em unidades de cuidado quimioterápico possibilitaram a observação de que o profissional que atua nessa área está exposto a riscos ocupacionais. Estes riscos podem ser do tipo biológico, físico, ergonômico, de acidentes e, principalmente, o risco químico, que está presente durante a manipulação dos agentes antineoplásicos, no contato direto destas drogas com pele e mucosas, na inalação de aerossóis, na ingestão de alimentos e de medicações contaminadas por resíduos desses agentes. ${ }^{7} \mathrm{Na}$ tentativa de reduzir os riscos ocupacionais, a biossegurança constitui um campo de conhecimento e um contínuo de práticas e ações técnicas, com preocupações sociais e ambientais, que são propostos para conhecer e controlar os riscos que o trabalho pode propiciar ao ambiente e à vida. ${ }^{8}$

Estas experiências ainda possibilitaram a observação de que os profissionais de Enfermagem têm dificuldade de perceber as condições capazes de gerar acidentes no decorrer de sua prática laboral. Portanto, a participação destes na identificação das condições causadoras de riscos ocupacionais é fundamental. ${ }^{7,9}$ 


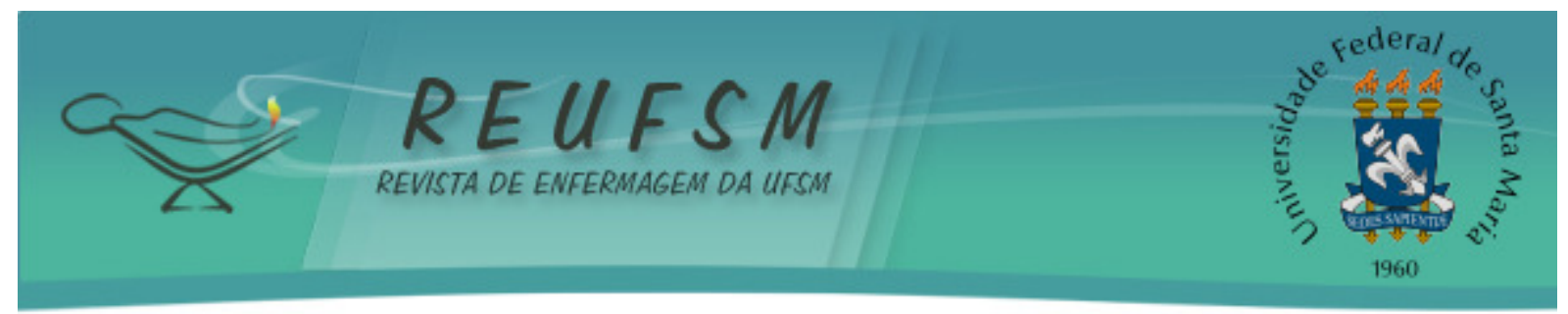

Diante disso, questiona-se: qual a percepção da equipe de Enfermagem sobre a biossegurança nos serviços de quimioterapia? 0 objetivo do estudo foi identificar a percepção dos profissionais de Enfermagem quanto a biossegurança no ambiente de cuidado quimioterápico.

\section{MÉTODO}

Trata-se de uma pesquisa de caráter exploratório-descritivo, a qual consiste em um estudo preliminar, que tem como principal objetivo familiarizar o pesquisador com o fenômeno a ser observado. ${ }^{10} \mathrm{~A}$ pesquisa descritiva é aquela que tem como função a análise do objeto de estudo por meio da observação, descrição e classificação dos fenômenos ocorridos. ${ }^{10-11}$

Foi realizado em um instituto de radioterapia e oncologia localizado no município de Uruguaiana, que se constitui no serviço de referência para atendimento de pacientes oncológicos da Região Oeste do Rio Grande do Sul, Brasil (RS).

A coleta dos dados foi realizada entre os meses de setembro e novembro de 2010 e ocorreu em dois momentos: o primeiro por meio de observação de campo não participante e o segundo por meio de entrevista semiestruturada. Os sujeitos da pesquisa foram dois enfermeiros e um técnico de enfermagem, que constituem a totalidade da equipe de Enfermagem do serviço em estudo.

As entrevistas foram realizadas pelos pesquisadores, gravadas em meio digital e transcritas. A palavra "Sujeito" foi utilizada seguida por números consecutivos para manter o anonimato dos participantes, uma vez que a equipe de Enfermagem do referido serviço é constituída por três trabalhadores. A Análise de Conteúdo ${ }^{12}$ subsidiou a análise dos dados, que foram organizados e categorizados com o auxílio do software NVivo $®$.

A pesquisa respeitou os princípios bioéticos postulados na Resolução 196/1996 ${ }^{13}$, tendo obtido a aprovação do Comitê de Ética em Pesquisa da Universidade Federal do Pampa (UNIPAMPA) sob o protocolo número 014/2010.

\section{RESULTADOS E DISCUSSÃO}

O tempo máximo de formação dos profissionais da Enfermagem do serviço pesquisado foi 25 anos e o mínimo dois anos. A média em anos de atuação em unidade quimioterápica foi nove anos, sendo que nenhum dos profissionais possui formação específica em Enfermagem oncológica ou áreas relacionadas.

Do processo de categorização dos dados emergiram duas categorias: a segurança do profissional em ambiente quimioterápico e a segurança do paciente em ambiente quimioterápico.

\section{A segurança do profissional em ambiente quimioterápico}

Os riscos ocupacionais dos profissionais da saúde, sobretudo da Enfermagem que se encontra diretamente envolvida na prestação do cuidado ao paciente com câncer, ocorre em qualquer momento do processo de trabalho, desde o preparo, a administração e o descarte dos quimioterápicos.

Os sujeitos demonstraram conhecimento acerca das medidas de biossegurança, a fim de evitar e/ou minimizar os possíveis acidentes no ambiente de trabalho. Revelaram ainda a preocupação do serviço em se adequar a legislação, no sentido de proporcionar segurança e qualidade ao profissional no atendimento prestado ao paciente, como pode ser observado nos seguintes relatos: 


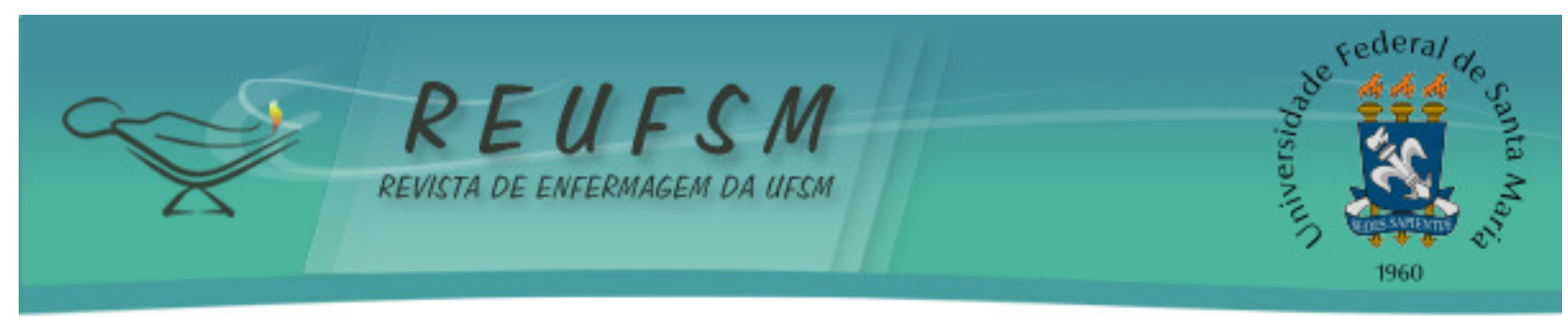

Dependendo do tipo de quimioterapia utiliza-se certos tipos de EPI. Além dos EPI tem os equipamentos de proteção ambiental [...], por isso que a gente sempre orienta os familiares a não permanecerem junto na sala acompanhando o paciente. Alguns pacientes, às vezes, têm a necessidade de ter um familiar junto, mas de preferência não, porque a quimioterapia [...] por mais que a gente faça ela em sistema fechado alguma coisa fica no ar, então por mais que agente usa a máscara, os familiares não e [por isso] quem entra numa sala de quimioterapia sente o cheiro. (Sujeito 1)

[...] máscara, avental, luva. A máscara é com filtro de carvão pra trabalhar na capela, embora essa não seja minha função, agora é função da farmacêutica [...] Eu até fazia antes, se precisasse, mas agora como ela veio ficou a cargo dela [...] quando iniciei não tinha a farmacêutica, nem a capela, e com o tempo acabou vindo a capela, a farmacêutica, está vindo enfermeiro e a informação está maior até porque veio o farmacêutico, o enfermeiro e agora temos vocês aqui [os alunos]... e antes não tinha enfermeiros eram só atendentes. (Sujeito 3)

No que concerne a percepção dos profissionais da Enfermagem quanto ao risco ocupacional, em 1970 a Occupational Safety and Health Administration (OSHA) definiu normas que garantissem o manuseio seguro dos citostáticos do ponto de vista pessoal e ambiental, sendo a capela de fluxo laminar vertical o mais importante elemento de proteção. ${ }^{2}$ Sobre a capela de fluxo laminar:

Aquela capela ali, ela está no lugar errado, mas ela não traz risco, porque o risco em si é alguém manusear ela erradamente, porque aquela capela de seis em seis meses... vem um profissional, não sei se é de São Paulo, é um técnico que vem e faz todas as medições e troca o filtro dela, também conforme toda a rotina da RDC que normatiza o serviço de quimioterapia [...] não deixar quimioterapia escapar a vazão, pra ela filtrar a quimioterapia. [...] a capela ia trazer um risco pra nos ali [...] se ela estivesse sendo usada errada $e$ se ela não tivesse essa manutenção necessária [...] e onde é controlado, porque tu imagina ela no lugar errado e ainda funcionando errado, ai ela estava "expelindo" quimioterapia junto conosco, por isso que a importância da capela ser preparada e sair o sistema fechado de dentro da capela, só usa quando vai usar no paciente, ai que eu digo para vocês da importância de a gente usar máscara quando está manuseando principalmente quando está fazendo uma medicação no paciente em bolus [...] que é nesse momento em que agente não enxerga, mas libera partículas aerossóis. (Sujeito 1)

Os profissionais do serviço demonstram ter conhecimento de que a capela de fluxo laminar está posicionada em local inadequado, pois no mesmo ambiente está o posto de enfermagem, armários para depósito de medicamentos, refrigerador de pequeno porte para armazenamento de medicamentos e lixeiras para o descarte dos resíduos do serviço. Apesar disso, foi relatado que a capela não traz risco para os profissionais e para os 


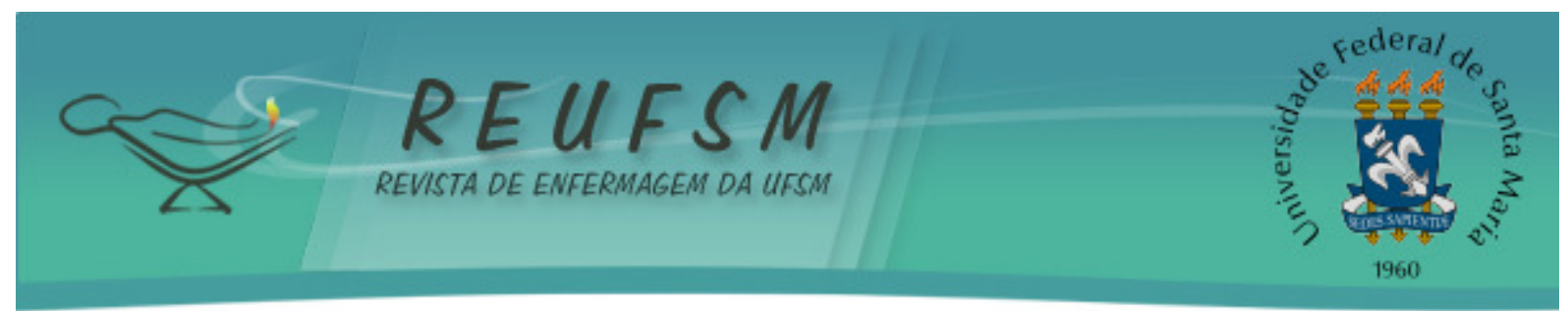

pacientes que circulam no ambiente. Conforme a RDC $n^{\circ} 220$ de 2004, deve existir uma sala exclusiva para preparação de medicamentos utilizados na terapia antineoplásica, com área mínima de cinco $\mathrm{m}^{2}$ por cabine de segurança biológica, sendo esta do tipo Classe II, de modo que o profissional não fique exposto às partículas dos antineoplásicos. ${ }^{14}$

Os profissionais do serviço identificam alguns riscos durante a administração de agentes antineoplásicos, porém não mencionam todas as precauções para evitar os riscos a que estão expostos. O cuidado no descarte de materiais visa prevenir danos aos profissionais responsáveis pela higienização do serviço, como pode ser observado nos relatos a seguir:

A nossa higienização é terceirizada. Antes não era, agora a gente terceiriza, mas existe toda normatização de como funciona: gerenciamento de resíduos, [que] também envolve a Enfermagem junto com a farmácia na oncologia. É um problema sério hospitalar, que a gente não dá muita importância pra isso, que a gente tem que se importar desde o momento que a gente produz até a finalização do resíduo, aonde que ele vai para a gente [...] ter ciência. (Sujeito 1)

[...] é tem que saber principalmente o pessoal da limpeza, que manipula o lixo [...] e aquele lixo é tóxico, gases né [...]. (Sujeito 3)

Observa-se que os sujeitos entrevistados têm consciência do descarte correto dos materiais a fim de prevenir acidentes, principalmente para os profissionais da higienização. Entretanto, foi observado que durante a troca de um quimioterápico houve o derramamento do mesmo no ambiente, sendo utilizado para limpeza um pano úmido que foi deixado sobre o local, medida que demonstra falta de adesão às normas sobre a limpeza de acidentes com quimioterápicos.

Os profissionais de Enfermagem durante a assistência ao paciente estão expostos a riscos ocupacionais causados por fatores químicos, físicos, biológicos, ergonômicos, de acidentes e psicossociais, que podem ocasionar doenças e/ou agravos ocupacionais ${ }^{15}$. Quanto à ocorrência de acidentes no serviço:

Não, não tive... o que a gente tem assim é, por exemplo, alguma reação adversa que não é costumeira da quimioterapia. (Sujeito 1)

Nunca, nem picada, nunca nada, e nem no hospital também eu acho que é o cuidado [...] a maioria dos funcionários que se pica é reencapando [agulhas] no horário entre seis e sete da manhã que é o maior fluxo de acidente de trabalho, horário que o funcionário já está cansado e está fazendo tudo rápido, é o horário das medicações. Ou é reencapando [agulhas], ou deixam solto na bandeja e se picam e é o maior fluxo da mania de reencapar... a gente tem que puncionar e largar na bandeja o mandril, mas nunca me piquei. (Sujeito2)

Não que eu lembre... acho que tiveram poucos até com os pacientes [...] não soube de ninguém, ninguém se picou nem nada... daí 


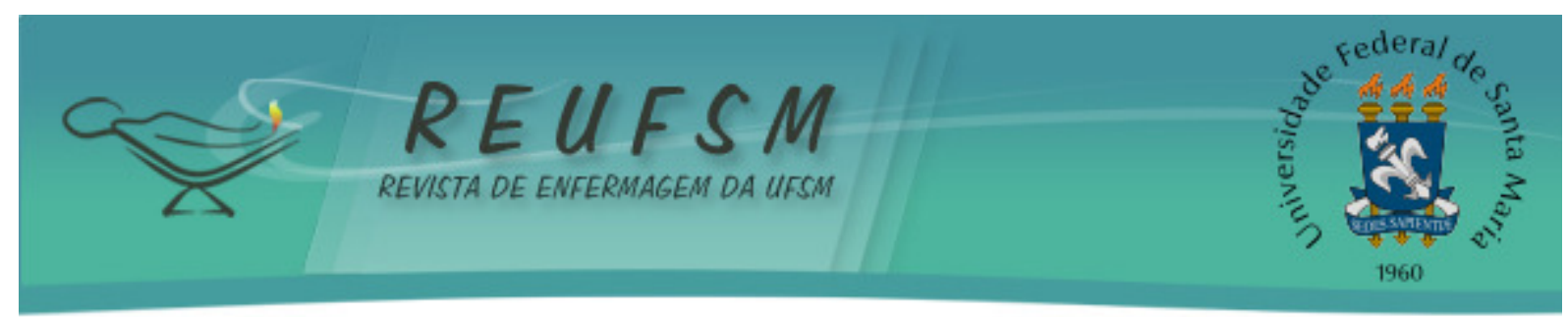

nesses anos que trabalho aqui não soube de nenhum acidente. (Sujeito 3)

Os sujeitos entrevistados relatam nunca terem sofrido acidentes de trabalho, sendo que um deles relata que apenas sofre acidente e/ou causa algum acidente o profissional que atua com a atenção diminuída. Um estudo demonstra que os maiores índices de acidentes no ambiente de saúde são pelo reencape de agulhas ${ }^{16}$, prática frequente no serviço pesquisado que expõe diariamente os profissionais a situações de risco de acidentes com material biológico e químico.

A manipulação de agentes antineoplásicos, na ausência de medidas de proteção adequadas, tem sido associada à absorção dos medicamentos entre a equipe de Enfermagem. Estudos realizados com profissionais que manipularam essas drogas evidenciaram casos de mutagenicidade celular, infertilidade, aborto e malformações congênitas, disfunções menstruais, bem como sintomas imediatos como tontura, cefaleia, náusea, alterações de mucosas e reações alérgicas. ${ }^{17-18}$

Para a manipulação dos antineoplásicos utilizam-se os EPI, conforme as instruções da RDC n 220 de 2004: luvas (tipo cirúrgica) de látex, punho longo, sem talco e estéreis; e avental longo ou macacão de uso restrito a área de preparação, com baixa liberação de partículas, baixa permeabilidade, frente fechada, com mangas longas e punho elástico. A paramentação, quando reutilizável, deve ser guardada separadamente, em ambiente fechado, até que seja lavada. 0 processo de lavagem deve ser exclusivo a este vestuário. ${ }^{14}$

$\mathrm{Na}$ observação realizada pelos pesquisadores, estas recomendações são parcialmente seguidas no serviço. Não ocorre a utilização do campo descartável na área de aplicação da quimioterapia, apenas é utilizada uma seringa e uma agulha para realizar aspiração da medicação antiemética. Este fato pode contribuir para a contaminação cruzada entre os pacientes, além do risco de acidentes durante o reencape das agulhas.

\section{A segurança do paciente em ambiente quimioterápico}

Apesar deste tema não ter sido foco do presente estudo, a segurança do paciente emergiu nas falas dos sujeitos merecendo atenção pela sua importância. A segurança do paciente visa a redução do risco de danos desnecessários associados à assistência em saúde até um mínimo aceitável. O “mínimo aceitável" consiste ao que é transitável diante do conhecimento atual, dos recursos disponíveis e da situação em que a assistência foi realizada visto o risco de não-tratamento, ou outro tratamento. ${ }^{19}$

O paciente submetido ao tratamento quimioterápico está exposto aos vários riscos como troca de protocolos na preparação das doses e na administração dos medicamentos, além da atuação indiscriminada e rápida das drogas antineoplásicas às células cancerosas e normais. O enfermeiro, que é o profissional habilitado para a administração de antineoplásicos, precisa conhecer seus pacientes através da realização do Histórico de Enfermagem a cada ciclo quimioterápico, devendo estar ciente do estado de saúde atual e possíveis riscos, danos e erros, preservando o paciente de possíveis eventos adversos. Essa conduta fica evidente nos trechos a seguir:

[...] o grande problema nosso da Enfermagem, infelizmente, é a toxidade das medicações [...] compromete muito a rede venosa do paciente e nesse comprometimento, às vezes, causa lesões nos vasos. (Sujeito 1) 


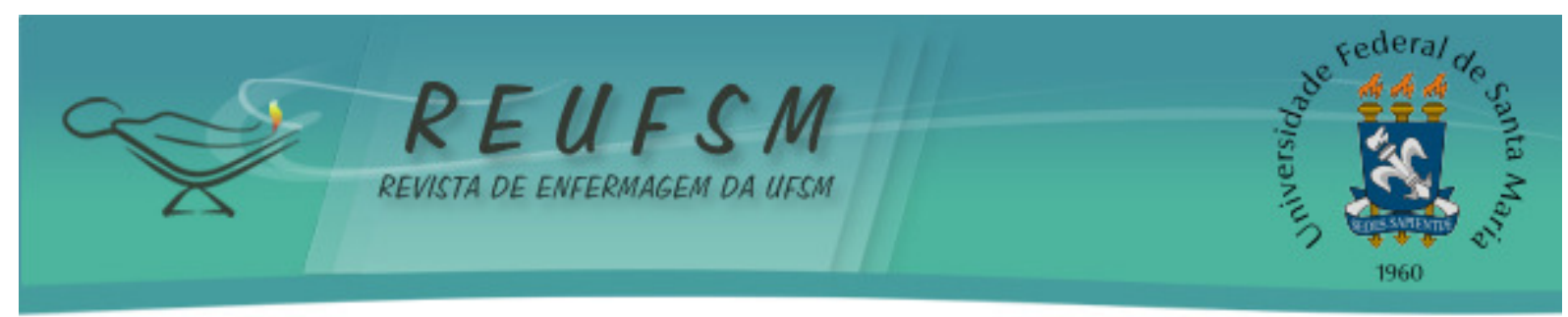

O que a gente tem assim é, por exemplo, alguma reação adversa que não é costumeira da quimioterapia [...] porque conforme você está administrando e o paciente apresentar um sintoma um sinal, tu tens que saber [o que a] medicação faz e tem esse tipo de sinal, já tem que sempre está um passo a frente, tem que orientar o paciente, 0 familiar e saber o cuidado que tu vai prestar. (Sujeito 2)

Observa-se que os sujeitos percebem somente os riscos não modificáveis no tratamento do paciente que faz uso de quimioterápico como o comprometimento da rede venosa. No entanto, não percebem os riscos modificáveis como o extravasamento de medicação que também pode causar lesões, assim como quando este ocorre no ambiente.

As medidas preconizadas pela literatura ao haver extravasamento de quimioterápicos consistem em, primeiramente, utilizar os Equipamentos de Proteção Individual (EPI) recomendados, remover o quimioterápico com o plástico absorvente desprezando-o identificado como lixo tóxico. A seguir, colocar sobre o local onde a medicação foi derramada solução hipoclorito de sódio a $1 \%$ embebida em uma compressa deixando-a agir por 15 minutos e realizar limpeza mecânica com detergente. ${ }^{2}$

Quanto ao risco da troca do medicamento quimioterápico:

[...] com quimioterapia, por exemplo, aqui se tu errar um medicamento num paciente no outro dia tu vai ver. Se tu usar num paciente medicação que não faz queda de cabelo e no outro dia o paciente vir sem cabelo [...]? Vão chegar em ti... tu vai dizer: não, eu fiz a medicação certa, e na realidade muitas vezes tu não fez, tu achou que fez [...] tem que simplesmente procurar diminuir os riscos de ter esse tipo de erro, rotular, cada prescrição é um paciente, identificar o paciente corretamente. E todo cuidado envolve também como a gente aprende na faculdade né: nome, dosagem, $e$ todas aquelas orientações que a gente recebe quando aprende farmacologia. [...] o principal é a atenção, responsabilidade do que tá fazendo, se tu tiver isso tu vai diminuir o risco infimamente, tu pode trabalhar com as coisas mais perigosas do mundo né, se tu prestar atenção, ter atenção no que tá fazendo e ter responsabilidade naquilo que tu faz dificilmente tu vai ter algum dano. (Sujeito 1)

É fundamental ter atenção com as doses e esquemas terapêuticos, uma vez que pequenas variações ou erros podem provocar danos letais ao paciente oncológico. Os profissionais envolvidos no preparo e na manipulação deverão certificar-se lendo e relendo a prescrição antes da manipulação, checar a dose recebida, principalmente quando o paciente recebe drogas com toxicidade cumulativa definida, ou seja, aquelas que se tornam proibitivamente tóxicas a determinados órgãos vitais, a partir de uma dose total predefinida. ${ }^{2}$ A técnica asséptica também deve sempre ser adotada na manipulação de quaisquer medicamentos para evitar possíveis infecções, conduta de suma importância no tratamento de pacientes oncológicos, uma vez que estes podem se encontrar imunodeprimidos seja pela doença ou pela própria droga antineoplásica.

O risco da ocorrência de iatrogenias está aumentado quando os serviços de saúde estão atendendo mais pacientes que o planejado e há carência de recursos humanos. A observação do serviço em questão demonstrou que os profissionais enfermeiros ficaram responsáveis pela administração de antineoplásicos de até 13 pacientes ao mesmo tempo. Nestes momentos, os profissionais colocam cadeiras entre os leitos e poltronas já existentes, o que dificulta a identificação dos pacientes. Além disso, os profissionais 


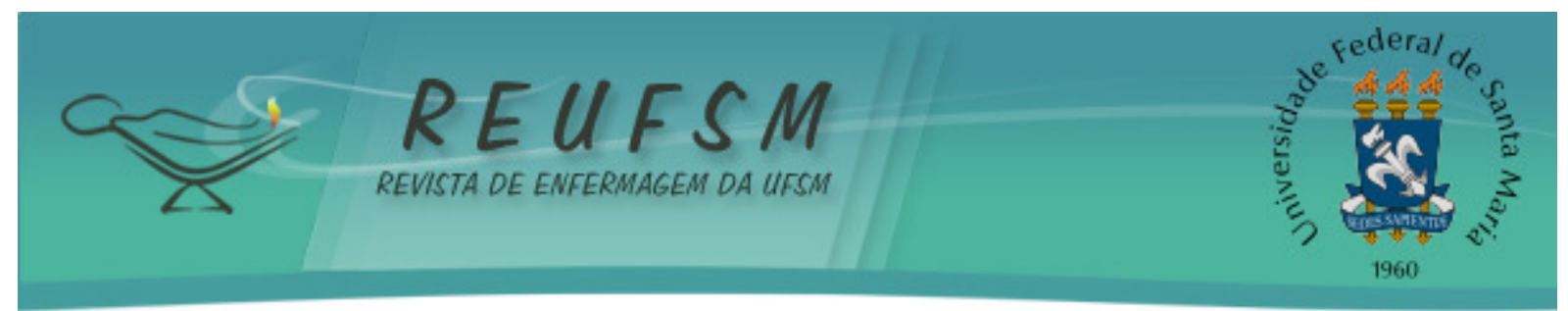

realizam jornadas duplas ou triplas de trabalho, visto que nenhum deles trabalha exclusivamente no serviço.

Para evitar erros durante a administração de medicamentos a Resolução 293 do Conselho Federal de Enfermagem estabelece parâmetros para o dimensionamento da equipe de Enfermagem, além da supervisão permanente dos técnicos de enfermagem pelo enfermeiro. ${ }^{20}$

\section{CONSIDERAÇÕES FINAIS}

A observação e as entrevistas realizadas com a equipe de Enfermagem permitiram identificar que os profissionais percebem alguns riscos a que estão expostos, porém não aderem às medidas de biossegurança no ambiente de cuidado quimioterápico. Esta constatação permite afirmar que é preciso conscientizar os profissionais quanto aos riscos na manipulação e administração de antineoplásicos, bem como qualifica-los para identificar riscos e utilizar medidas de proteção.

As práticas adotadas pela equipe de Enfermagem do serviço os expõem ao risco de contato direto com as drogas antineoplásicas e a inalação de partículas aerossolizadas, além de favorecer a contaminação do ambiente de trabalho. Porém, os profissionais não percebem como risco o fato de estarem expostos a drogas e à inalação de partículas pela capela de fluxo laminar não estar em uma sala exclusiva para preparação de medicamentos. Quanto aos acidentes, ao reencapar agulhas, os profissionais o reconhecem, mas não relataram preocupação com relação a utilizar a mesma seringa e agulha para administrar medicação nos pacientes que são atendidos no serviço. Para evitar acidentes e a contaminação cruzada de pacientes, é recomendado o descarte de agulhas e seringas imediatamente após o uso e sem reencapá-las.

Diante do exposto, afirma-se a necessidade da realização da educação permanente para garantir a segurança do profissional que atua nos serviços de quimioterapia. É preciso que este tenha conhecimento específico para trabalhar com quimioterápicos, pois a atividade requer alta especialização e constante atualização. Espera-se que esta pesquisa contribua para conscientizar os profissionais destes serviços sobre a importância das medidas de biossegurança, assim como alerte os gestores sobre os riscos e a necessidade de oferecer condições de trabalho e ambiente seguros tanto para os profissionais quanto para os pacientes oncológicos.

\section{REFERÊNCIAS}

1. McGuinn LA, Ghazarian AA, Ellison GL, Harvey CE, Kaefer CM, Reid BC. Cancer and environment: definitions and misconceptions. Environmental Res. 2012 Jan;112:230-4.

2. Bonassa EMA, Santana TR. Enfermagem em terapêutica oncológica. São Paulo: Atheneu; 2005.

3. Mohallem AGC, Rodrigues AB. Enfermagem oncológica. São Paulo: Manole; 2007.

4. Siegel R, Desantis C, Virgo K, Stein K, Mariotto A, Smith T, et al. Cancer treatment and survivorship statistics, 2012. CA Cancer J Clin. 2012;62(4):220-41.

5. Siegel R, Naishadham D, Jemal A. Cancer statistics, 2013. CA Cancer J Clin. 2013;63(1):11-30.

6. Brasil. Ministério da Saúde. Secretaria de Atenção à Saúde. Instituto Nacional de Câncer (INCA). A situação do câncer no Brasil. Rio de Janeiro: INCA; 2006.

7. Castro MR, Farias SNP. A produção científica sobre riscos ocupacionais a que estão expostos os trabalhadores de enfermagem. Esc Anna Nery Rev Enferm. 2008;12(2):364-9. 


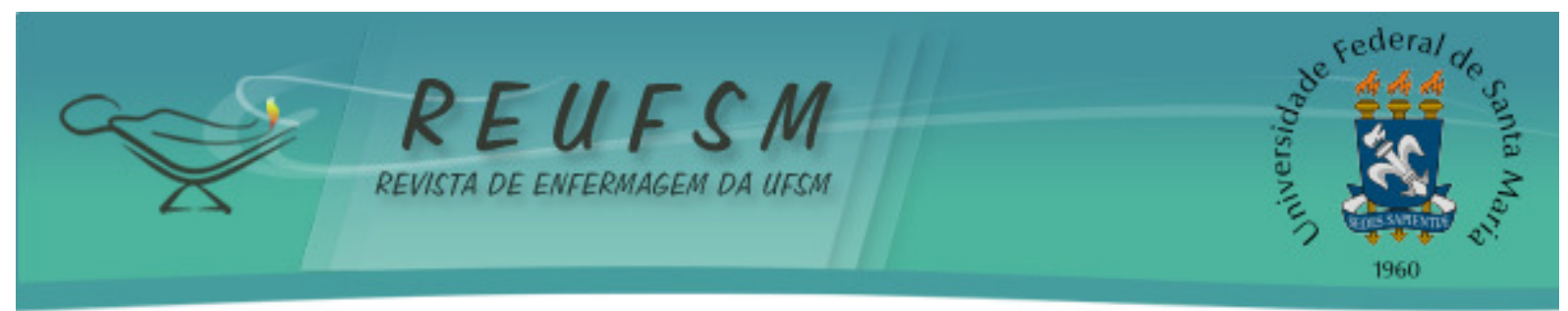

8. Cararro TE, Gelbcke FL, Sebold LF, Kempfer SS, Zapelini MC, Waterkemper R. Nursing students' point of view on biosecurity and patient safety. Rev Gaúch Enferm. 2012 Sep;33(3):14-9.

9. Cummings G, Olson K, Raymond-Seniuk C, Lo E, Masaoud E, Bakker D, et al. Factors influencing job satisfaction of oncology nurses over time. Can Oncol Nurs J. 2013;23(3):162-81.

10. Neves JL. Pesquisa qualitativa: características usos e possibilidades. Caderno de Pesquisa em Administração (São Paulo). 1996;1(3):1-5.

11. Walker JL. The use of saturation in qualitative research. Can J Cardiovasc Nurs. 2012;22(2):37-46.

12. Bardin L. Análise de conteúdo. Lisboa (Portugal): Edições 70; 1977.

13. Brasil. Ministério da Saúde. Conselho Nacional de Saúde. Resolução CNS n 196, de 10 de outubro de 1996. Aprovar as seguintes diretrizes e normas regulamentadoras de pesquisas envolvendo seres humanos. Brasília (DF): Ministério da Saúde; 1996.

14. Brasil. Ministério da Saúde. Agência Nacional de Vigilância Sanitária. Resolução RDC $n^{\circ} 220$, de 21 de setembro de 2004. Aprova o Regulamento Técnico de funcionamento dos Serviços de Terapia Antineoplásica. Diário Oficial da União, Brasília; 2004 set 23. Seção 1, p. 72-5.

15. Sulzbacher E, Fontana RT. Concepções da equipe de enfermagem sobre a exposição a riscos físicos e químicos no ambiente hospitalar. Rev Bras Enferm. 2013 fev;66(1):25-30.

16. Rogowska-Szadkowska D, Stanislawowicz M, Chlabicz S. Risk of needle stick injuries in health care workers: bad habits (recapping needles) last long. Przegl Epidemiol. 2010;64(2):293-5.

17. Friese CR, Himes-Ferris L, Frasier MN, McCullagh MC, Griggs JJ. Structures and processes of care in ambulatory oncology settings and nurse-reported exposure to chemotherapy. BMJ Qual Saf. 2012 Sep;21(9):753-9.

18. Fassini $\mathrm{P}$, Hahn $\mathrm{GV}$. Riscos à segurança do paciente em unidade de internação hospitalar: concepções da equipe de enfermagem. Rev Enferm UFSM. [acesso em 2013 abr 10] 2012;2(2):290-9. Disponível em: http://cascavel.ufsm.br/revistas/ojs-

2.2.2/index.php/reufsm/article/view/4966/3753

19. World Health Organization (WHO). Conceptual framework for the International Classification for Patient Safety (v.1.1) - Final Technical Report and Technical Annexes. WHO; 2009.

20. Conselho Regional de Enfermagem. Resolução COFEN $n^{\circ}$ 293/04. Fixa e estabelece parâmetros para dimensionamento do quadro de profissionais de enfermagem nas instituições de saúde [Internet]. São Paulo: Conselho Federal de Enfermagem; 2004 [acesso em 2013 nov 14]. Disponível em: http://novo.portalcofen.gov.br/wpcontent/uploads/2012/03/RESOLUCAO2932004.PDF.

Data de recebimento: 04/04/2013

Data de aceite: 31/03/2014

Contato com autor responsável: Wendel Mombaque dos Santos

Endereço postal: Rua 14 de julho, 2790, apartamento 201. CEP: 97510-450 - Uruguaiana/RS

E-mail: wendelmombaque@hotmail.com 